\title{
Gastric outlet obstruction with ascites: EUS-guided gastro-enterostomy is feasible
}

\section{(ㄷ)(1) $\odot$}

\section{Authors}

Jahangeer Basha, Sundeep Lakhtakia, Raghavendra Yarlagadda, Zaheer Nabi, Rajesh Gupta, Mohan Ramchandani, Radhika Chavan, Nitin Jagtap, Shujaath Asif, Guduru Venkat Rao, Nageshwar Reddy

Institution

Asian Institute of Gastroenterology, Hyderabad, India

submitted 23.6.2021

accepted after revision 23.8.2021

Bibliography

Endosc Int Open 2021; 09: E1918-E1923

DOI 10.1055/a-1642-7892

ISSN 2364-3722

(C) 2021. The Author(s).

This is an open access article published by Thieme under the terms of the Creative Commons Attribution-NonDerivative-NonCommercial License, permitting copying and reproduction so long as the original work is given appropriate credit. Contents may not be used for commercial purposes, or adapted, remixed, transformed or built upon. (https://creativecommons.org/licenses/by-nc-nd/4.0/)

Georg Thieme Verlag KG, Rüdigerstraße 14,

70469 Stuttgart, Germany

\section{Corresponding author}

Sundeep Lakhtakia, MD, DM, Director Endoscopy \& EUS, Asian Institute of Gastroenterology, 6-3-661 Somajiguda

Hyderabad, Hyderabad, Telangana 500081, India

Fax: +91-40-23324255

drsundeeplakhtakia@gmail.com

\section{ABSTRACT}

Background and study aims Endoscopic ultrasoundguided gastro-enterostomy(EUS-GE) is a recently described novel minimally invasive endoscopic procedure for patients having malignant gastric outlet obstruction (GOO). The safety of EUS-GE in the presence of ascites with GOO is not known. The objective of the study was to evaluate the feasibility and safety of EUS-GE in patients with GOO and ascites.

Patients and methods Consecutive patients with GOO who underwent EUS-GE between January 2019 and March 2021 constituted the study population. EUS-GE was performed using either EPASS or free-hand technique. The technical success, clinical success, adverse events, and survival times were evaluated. The outcomes were compared between patients with and without ascites.

Results A total of 31 patients with GOO underwent EUS-GE of whom 29 (93.5\%) had malignant and two (6.4\%) had benign etiologies. Ascites was observed in 12 out of 31 (38.7\%) patients and all had underlying malignancy. Majority $(27,87 \%)$ of the EUS-GE procedures were performed using EPASS technique, and 4 (13\%) underwent free-hand technique. Eleven of 12 patients with ascites and GOO underwent EUS GE using EPASS technique. The technical success $(91.6 \%$ vs. $89.4 \% ; P=0.841)$, clinical success $(83.3 \%$ vs. $89.4 \% ; P=0.619$ ), mean procedure time (32 vs. $31.6 \mathrm{~min}$; $P=0.968)$ and adverse events ( $0 \%$ vs. $10.5 \% ; P=0.245$ ) were not significantly different between patients with or without ascites. However, the median survival time was significantly low in patients with ascites when compared to without ascites ( 36 vs. 290 days; $P<001$ ).

Conclusions Ascites is a common occurrence in patients with malignant GOO. EUS GE is feasible in presence of ascites with EPASS technique.

\section{Introduction}

Endoscopic ultrasound guided gastro-enterostomy (EUS-GE) is a novel procedure, recently described for patients with gastric outlet obstruction (GOO) [1,2]. The reported technical and clinical success of this procedure is high $[3,4]$. Ascites is often observed in $\mathrm{GOO}$ patients due to progression of malignancy. EUS-GE in presence of ascites carries theoretical risk of adverse events, especially perforation, where the fallback option is often surgery. The feasibility and safety of EUS-GE in patients with GOO with ascites has not been objectively addressed in literature. There are several described methods of doing EUS-GE, whose preference is based on operator experience and availability of devices [5]. The technical challenges and adverse events of EUS-GE in presence of ascites are not well known. The objective of this study was to evaluate the feasibility and safety of EUS-GE in patients with gastric outlet obstruction and ascites. 

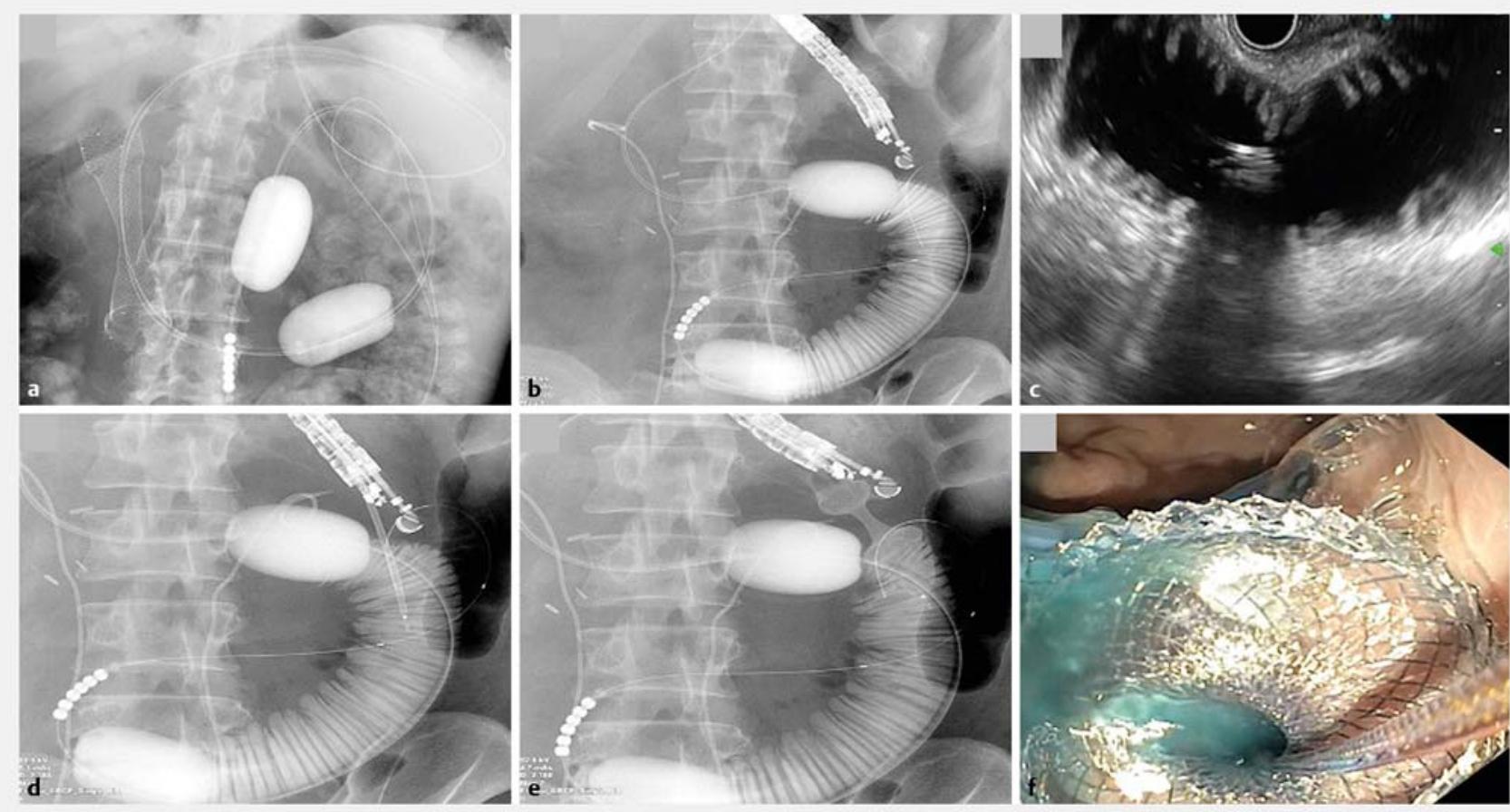

- Fig. 1 EUS-guided gastroenterostomy using the EPASS technique. a Fluoroscopic image showing the EPASS catheter passing across the stricture with inflated both proximal and distal balloons and the previously placed blocked enteral SEMS and biliary SEMS. b Fluoroscopic image showing EUS scope targeting vertical limb of contrast filled jejunal segment between the two balloons. c EUS image showing the distended jejunal segment. EPASS catheter can be seen in the lumen. $\mathbf{d}$ Fluoroscopic image showing EUS scope and puncture of jejunal segment with Hot Axios delivery system. e Fluoroscopic image showing deployed LAMS between jejunum and stomach. $\mathrm{f}$ Endoscopic image showing deployed LAMS draining coloured contrast fluid. EPASS, EUS-guided double-balloon-occluded gastrojejunostomy bypass; SEMS, self expandable metal stent; LAMS, lumen apposing metal stent.

\section{Patients and methods}

Consecutive patients with symptomatic GOO who underwent EUS GE between January 2019 to March 2021 constituted the study population. The study was conducted at a tertiary care hospital and approved by institutional ethical committee. Demographic details, aetiology of $\mathrm{GOO}$ and presence of ascites during evaluation were recorded. Patients with ascites were further evaluated by ascitic fluid analysis including the cytology to establish the cause of ascites. In patients with malignant ascites, distal intestinal or multi-level obstruction was excluded by contrast imaging. Ascites was managed according to the standard of care. Patients with high SAAG (Serum Ascites Albumin Gradient) ascites, were managed by diuretics and intravenous albumin when required. Patients with malignant ascites were considered for paracentesis whenever they were symptomatic. In patients with significant ascites, either large volume paracentesis or percutaneous catheter ( $12 \mathrm{Fr}, 25 \mathrm{~cm}$ drainage catheter, Cook Medical, USA) placement for drainage was considered. Paracentesis was performed on the same day before the procedure. Catheter drainage in patients with rapidly filling ascites was placed a day before the procedure.

\section{Procedure}

EUS-GE was performed either by double balloon assisted (EPASS technique) or free-hand technique. All patients received intra- venous antibiotics prior to the procedure. The procedure was performed under anaesthetist supervised sedation without endotracheal intubation.

\section{EPASS technique}

A clean stomach devoid of any food residue was ensured by prior endoscopy or lavage through nasogastric tube. The patient was placed in either prone or supine position.

The steps in the procedure were as follows:

1. A standard gastroscope was passed through the over-tube of short enteroscope. The whole assembly was inserted into the stomach as one unit. The tip of the over-tube was kept close to the stricture (for gastric cancer) or in duodenal cap (for duodenal stricture).

2. A long $(450 \mathrm{~cm})$ guidewire was passed through catheter and negotiated across the stricture deep inside the proximal jejunum.

3. The gastroscope was removed, leaving the over-tube and guidewire in place. The purpose of over-tube was to prevent looping of the guidewire and/or the flexible soft oro-enteric tube having double balloon (EPASS) in stomach.

4. The EPASS balloon catheter was passed under fluoroscopy over the guidewire through the over-tube, across the luminal stricture, deep into proximal jejunum. The radio-opaque markers located at the tip and at the two balloons help in its accurate positioning ( $\vee$ Fig. $1 \mathbf{a})$. 


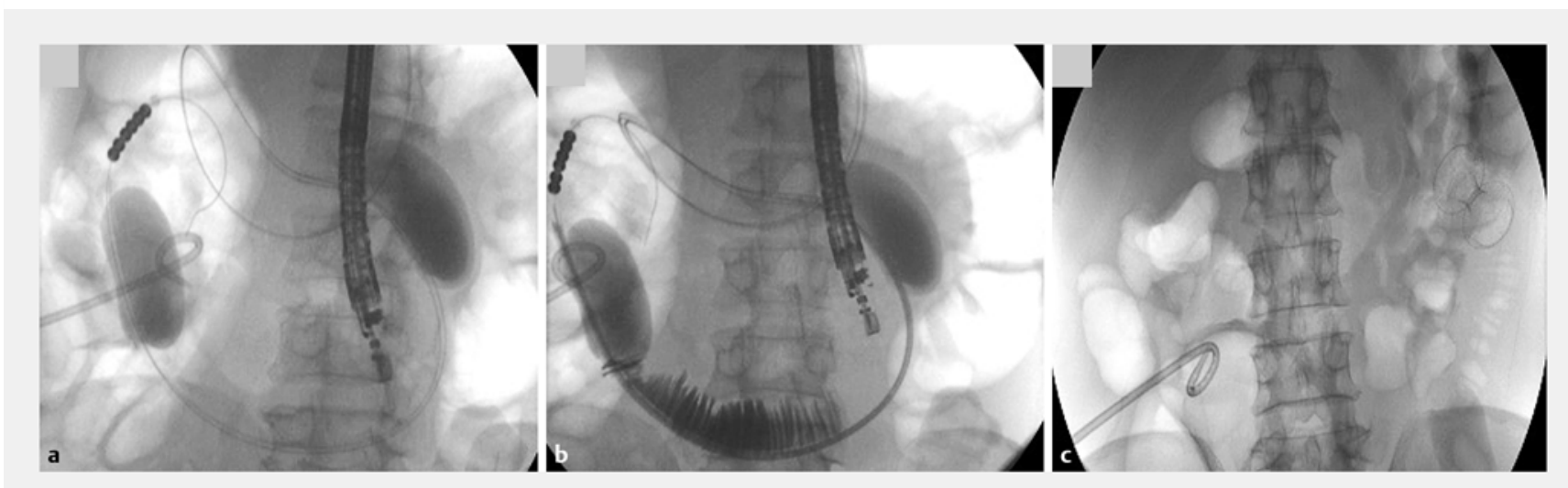

Fig. 2 EUS-guided gastroenterostomy in a patient with ascites. a Fluoroscopic image showing EUS scope and EPASS catheter passing across the stricture with inflated both proximal and distal balloons and the percutaneous catheter placed to drain the ascites. b Fluoroscopic view of EUS scope and contrast-injected jejunal segment between the two balloon. c Fluoroscopic image showing deployed successfully deployed LAMS. EPASS, EUS-guided double-balloon-occluded gastrojejunostomy bypass; LAMS, lumen apposing metal stent.

5. The two balloons (front and back) were then distended with about 40cc of diluted contrast to appose and anchor the small bowel loops ( $\triangleright$ Fig. 1a, $>$ Fig. 2a).

6. The intervening proximal small bowel (jejunal) segment between the 2 balloons was filled and distended with 100$120 \mathrm{ml}$ solution consisting of saline, contrast and a colouring agent (indigo-carmine) ( $>$ Fig. 1b, $\triangleright$ Fig. 2b).

The double balloon (EPASS) catheter has four dedicated channels (one for distal balloon, one for proximal balloon, one for the guidewire, and one for the filling the bowel segment between the two balloons. The aim was to place the proximal balloon, identified by respective radio-opaque marker, near DJ flexure and distal balloon beyond duodeno-jejunal flexure.

With the free-hand technique, either a standard feeding enteral tube (12 Fr, $120 \mathrm{~cm}$, Fresennius Kabi, Germany) or a biliary catheter (6 Fr, $250 \mathrm{~cm}$, Meditech, India) was passed through mouth over the guidewire across the luminal stricture segment into proximal jejunum. The target jejunal loop was distended rapidly with normal saline mixed with contrast and / or colouring agent under EUS and fluoroscopy vision. In cases with Afferent loop obstruction, guidewire was used after puncture with $19 \mathrm{~g}$ needle of the adherent distended afferent loop.

A linear echoendoscope (GF-UCT180; Olympus) was passed into the stomach at the time of filling the target jejunum lumen. The selectively distended jejunal segment, preferably the descending vertical limb, was identified under EUS and crosschecked on fluoroscopy before puncture ( $\triangleright$ Fig.1c). EUS-GE was performed using a 20- or 15-mm lumen apposing metal stent (LAMS) (HOT Axios, Boston Scientific, Marlborough, Massachusetts). The distal flange of the LAMS was deployed first under EUS and fluoroscopic guidance (ฉ Fig.1d, $>$ Fig.1e, - Fig. 2c). The proximal flange was then deployed either within the channel or under endoscopic view. The appearance of blue colouring agent in gastric lumen confirms the accurate placement of LAMS ( $>$ Fig. 1f). Often the pink mucosa of the small bowel was visible through the stent. Balloon dilation of the stent was not performed. The LAMS gradually expands over the next few days.

Patients were kept nil by mouth on the day of the procedure. A screening oral contrast study was performed at 24 to 48 hours to confirm the path of gastric emptying. Oral liquids were allowed after the contrast study, followed by low-residue feeds on subsequent day. Antibiotics were continued for 5 days in patients with ascites. Patients were discharged when an oral semisolid diet was well tolerated and if they were clinically stable. Rates of technical success, clinical success, and adverse events (AEs) were evaluated. The outcomes were compared between patients with and without ascites. All patients were followed every month, till death or last contact (Physical or by Telephone).

\section{Definitions}

Technical success was defined as successful deployment of LAMS between the proximal small bowel (jejunum or distal duodenum) and the stomach. Clinical success was defined as relief of symptoms of GOO and ability to tolerate diet.

Ascites was defined as mild, moderate, or large, according to definitions given by International ascites club [6]. Ascites was mild if it was detectable only on ultrasound. Moderate ascites caused moderate symmetrical distension of the abdomen. Large ascites caused marked abdominal distension

\section{Statistical analysis}

Categorical and continuous data were expressed as proportion and mean (standard deviation [SD]), respectively. All categorical variables were compared using a chi-square test or Fisher exact test wherever required. Continuous data were compared using an independent $t$ test. The survival of patients with or without ascites was compared with Kaplan-Meier analysis and log-rank test. $P<0.05$ was considered statistically significant. Statistical analysis was done using SPSS version 26 (IBM Corp., Armonk, New York, New York, United States) 
- Table 1 Comparison of clinical profile and outcomes between patients of gastric outlet obstruction with and without ascites.

\begin{tabular}{|c|c|c|c|}
\hline & $\begin{array}{l}\text { GOO with ascites } \\
\mathrm{N}=12(38.7 \%)\end{array}$ & $\begin{array}{l}\text { GOO without } \\
\text { ascites } N=19(61.3 \%)\end{array}$ & $P$ value \\
\hline Age in years, mean \pm S.D. & $62.2 \pm 12.5$ & $61.2 \pm 9.6$ & 0.806 \\
\hline Sex (male) & $8(66.6 \% \%)$ & $12(63.1 \%)$ & 0.842 \\
\hline Etiology of GOO, n (\%) & & & 0.481 \\
\hline Pancreatic cancer (15) & $6(50 \%)$ & $9(47.3 \%)$ & \\
\hline Periampullary cancer (2) & $2(16.6 \%)$ & $0(0 \%)$ & \\
\hline Gall bladder cancer (5) & $2(16.6 \%)$ & $3(15.7 \%)$ & \\
\hline Duodenal cancer ( 1 ) & $0(0 \%)$ & $1(5.2 \%)$ & \\
\hline Gastric cancer (4) & $1(8.3 \%)$ & $3(15.7 \%)$ & \\
\hline Postoperative recurrence in GJ anastomotic site (2) & $1(8.3 \%)$ & $1(5.2 \%)$ & \\
\hline Benign (2) & $0(0 \%)$ & $2(10.5 \%)$ & \\
\hline Procedural technique & & & 0.546 \\
\hline EPASS & $11(91.6 \%)$ & $16(84.2 \%)$ & \\
\hline Free hand & $1(8.3 \%)$ & $3(15.7 \%)$ & \\
\hline Technical success & $11(91.6 \%)$ & $17(89.4 \%)$ & 0.841 \\
\hline Procedure time in minutes (mean $\pm S D$ ) & $32 \pm 12.5$ & $31.6 \pm 6.6$ & 0.968 \\
\hline Clinical success & $10(83.3 \%)$ & $17(89.4 \%)$ & 0.619 \\
\hline Adverse events & $0(0 \%)$ & $2(10.5 \%)$ & 0.245 \\
\hline Median survival (days) & 36 & 290 & 0.001 \\
\hline
\end{tabular}

\section{Results}

A total of 31 patients underwent EUS-GE. Approximately twothirds (20 of 31 ) were male. The mean age was 61.65 years \pm 10.65 (SD). The etiology of GOO was malignancy in 29 patients (93.5\%), two patients had benign causes (duodenal obstruction due to chronic pancreatitis in one, retroperitoneal fibrosis in one patient). Among these, prior enteral SEMS were in situ and blocked in three patients due to tumor ingrowth. Prior biliary SEMS were present in six patients (ERCP 4, EUS guided choledocho-duodenostomy 2). Three patients underwent EUS-guided hepatico-gastrostomy (HGS) for biliary obstruction following EUS-GE, at the same session.

Overall, ascites was observed in 12 of 31 patients (38.7\%) prior to EUS-GE. Among the patients with malignant GOO, ascites was present in $41.3 \%$ (12 of 29). The mean age, sex, and etiology of GOO were not significantly different between patients with or without ascites ( $>$ Table 1). Ascitic fluid evaluation revealed cytology positive for malignancy in four patients. The remaining eight patients with negative cytology had high a high serum ascites albumen gradient (chronic liver disease in 1 , portal vein thrombosis in 1 , liver metastasis in 2 and hypo-albuminemia in 4 ). The ascites was mild to moderate in five patients. The remaining seven patients had large ascites, three of whom underwent therapeutic paracentesis once before the procedure. A per-cutaneous catheter was placed in the other four patients. The catheter was removed in one patient 3 days after EUS-GE and in the remaining three patients, catheters were left in situ.

\section{Technical success}

EPASS technique was used in 27 patients ( $87 \%$. Four (13\%) underwent the free-hand technique. Overall, EUS-GE was successfully performed in 28 of 31 patients (90.3\%). The mean procedure time was 31.88 minutes \pm 10.13 (SD). There was no difference in technical success between patients with or without ascites $(91.6 \%$ vs. $89.4 \%$; $P=0.841)$. Also, the mean procedure time was not significantly different (32 vs. $31.6 \mathrm{~min} ; P=0.968$ ).

LAMS placement was unsuccessful in three patients ( 2 with EPASS and one free-hand). This occurred in two patients in our early phase of LAMS experience while using the EPASS technique and both patients had only gastric (without jejunal) puncture. The gastric defect was then closed with an over-thescope-clip (OTSC) followed by enteral SEMS placement in the same session. The third patient underwent LAMS placement using the free-hand technique and developed colonic perforation requiring surgery.

During EUS-GE with EPASS, unexpected technical challenges were encountered in seven patients during the procedure. In two patients, there was distal seepage of contrast saline-colored solution trapped between the two anchoring balloons due to suboptimal anchoring leading to incomplete occlusion of the lumen by the forward balloon. The event was identified before the LAMS deployment, although it caused some delay. 
In another two patients, even after full inflation, the balloons migrated proximally due to friction caused by echoendoscope movement, making the procedure difficult. In another patient, duodenal narrowing was located in the distal duodenum near the DJ flexure, hence both balloons were positioned beyond the DJ flexure, causing an atypical location of the target jejunal segment, leading to difficulty in deployment of LAMS. Malrotation of the duodenum or location of the DJ flexure on the right side was observed in two patients. This caused awkward positioning of the echoendoscope at puncture. In spite of the above technical challenges, the EUS-GE was technically successful in all.

\section{Clinical success}

Overall, clinical success was achieved in 27 patients (87\%). One patient with postoperative recurrence of malignancy in a gastro-jejunostomy anastomotic site had persistent symptoms after LAMS placement in an obstructed afferent limb. On reevaluation, endoscopy confirmed efferent loop stoma narrowing due to the recurrence. Hence, an enteral SEMS was placed into the efferent limb after 2 days and his overall symptoms improved. Clinical success was similar in patients with and without ascites (83.3\% vs. $89.4 \%$; $P=0.619$ ).

\section{Adverse events}

AEs were observed in two patients (6.4\%). One had colonic perforation requiring surgery while using the free-hand technique. Another patient with benign duodenal stricture due to chronic pancreatitis and portal vein thrombosis with peri-gastric collaterals had gastrointestinal bleeding during LAMS deployment [7]. Gastric side bleeding was controlled with clips and jejunal side bleeding was controlled by passing the gastroscope through the LAMS and applying the clip over jejunal puncture side. Both these AEs occured in patients without ascites; however, the difference was not statistically significant (0\% vs. $10.5 \% ; P=0.245)$. None of the patient with ascites had infection or peritonitis after the procedure.

\section{Follow-up}

Twenty two of 28 patients (78.5\%) with successful LAMS placement died at a median follow-up of 66 days (range: 8 to 417 days). One patient with postoperative recurrence of cancer in an anastomotic site died 8 days after the procedure due to underlying cardiac disease. Of the two patients with benign GOO, one with retroperitoneal fibrosis died at 85 days after EUS-GE due to multisystem involvement and renal failure. Overall, the median survival time after the procedure was 51 days (range: 8 to 417 days). The median survival time in patients with ascites was 36 days (range: 8 to 180 days), whereas in patients without ascites, it was 290 days (range: 15 to 417 days). On Kaplan-Meier analysis, the difference in median survival was statistically significant (36 vs. 290 days; $P<0.001$ ) ( Fig. 3 ).

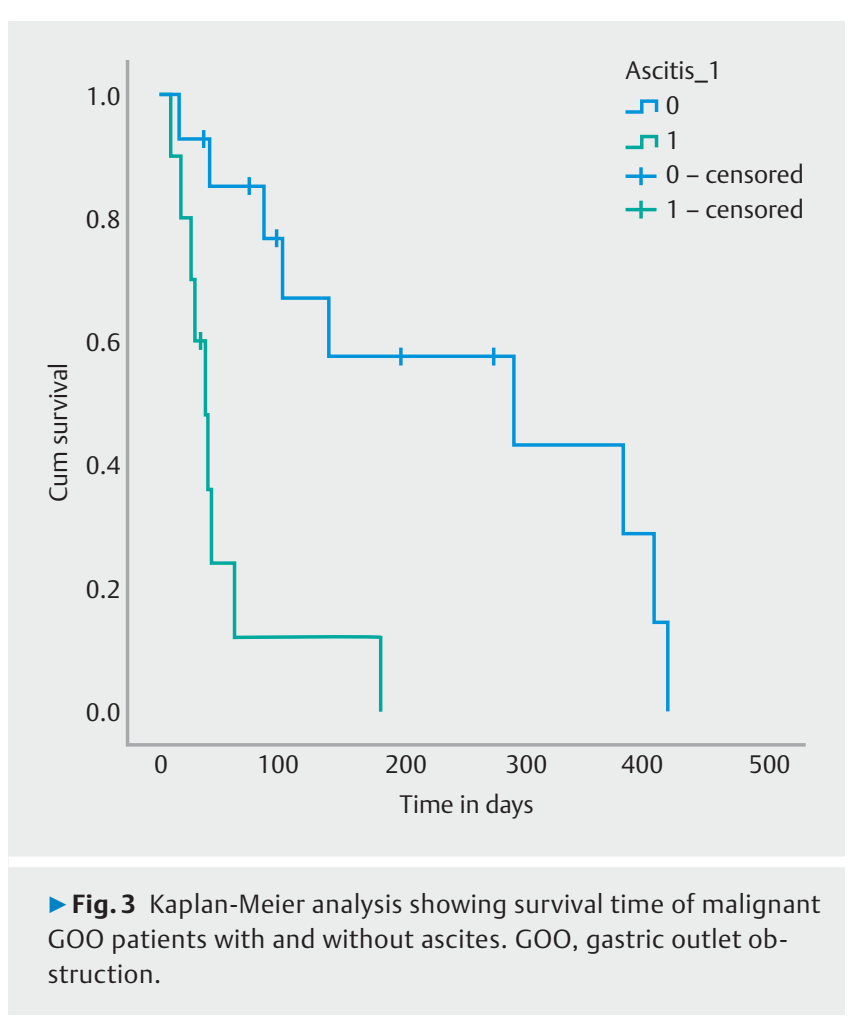

\section{Discussion}

Preferred palliative management for malignant GOO is surgical gastrojejunostomy in suitable patients, which is associated with long-term patency. Presence of ascites in these patients makes them unsuitable for surgical G]. Endoscopic enteral stent placement is an alternate option in surgically ineligible patients because of its minimal invasive nature [8]. The major drawback of enteral SEMS is tumor ingrowth associated with recurrence of GOO. EUS-GE is a recent, exciting alternate endoscopic option that appears to be feasible and safe in expert hands with good technical and clinical success $[3,4]$. Philip and colleagues recently compared EUS GE with enteral SEMS and reported higher clinical success and lower stent occlusion rates with EUS-GE [9]. The major benefit of EUS-GE over enteral SEMS is that the gastro-enterostomy stent is physically away from the tumor, and hence, less susceptible to malignant tumor ingrowth and occlusion. In the current study, we report that EUS-GE can be safely performed in patients with GOO even in the presence of ascites, after taking reasonable precautions.

Several techniques have been described for performing EUS$G E$, including free-hand, assisted (balloon, basket), and EPASS [10] double balloon. A recent retrospective comparative study showed that the direct free-hand and balloon-assisted techniques have comparable technical and clinical success rates [11]. However, there have been no direct comparative studies of those assisted techniques with EPASS. In this study, we used EPASS in the majority of patients and the overall technical and clinical success rateswere comparable to previous publications $[4,10,11]$. 
Ascites is not uncommon in malignant $\mathrm{GOO}$ and is generally associated with poor prognosis. Ascites was observed in nearly $40 \%$ of patients with malignant GOO in the current study. Pathophysiology of ascites in malignant GOO is multifactorial, including peritoneal involvement by malignancy, malnutrition, and portal hypertension due to underlying cirrhosis or liver metastases. The presence of ascites poses challenges for the procedure and anesthesia. Significant ascites precludes close apposition of the freely mobile jejunum to the stomach, thus posing a technical challenge for EUS-GE. To reduce the effect of ascites on EUS-GE, especially when there is large ascites, drainage of the ascites prior to the procedure may be required. We carefully evaluated and assessed ascites before performing EUS-GE. Wherever we felt significant ascites, we drained it with paracentesis or a catheter. Such precautions prevented any interloop fluid stagnation requiring aspiration during the EUS-GE. Irani et al described placement of LAMS in presence of ascites before gallbladder drainage and afferent loop obstruction [12].

Our study is the first to describe EUS-GE in the presence of ascites with comprehensive details of the patients with GOO and ascites and their management.

In this study, EPASS was used in the majority of GOO patients. We believe that the double balloon technique helps to anchor and fix the jejunal loop, thus allowing ease of the final step of LAMS placement. The stability even in the presence of ascites provides additional safety of the procedure. Almost all procedures in $\mathrm{GOO}$ with ascites were performed exclusively using EPASS except one with afferent loop obstruction. There were no technical failures or AEs due to ascites per se. Peritoneal infection is an expected complication in these patients. However, none of the patients developed any evidence of it immediately after the procedure or during follow-up, which could be due to adequate prophylactic antibiotic coverage before the procedure. In this study, the median survival was low in patients with ascites when compared to those without ascites Presence of ascites generally indicates an advanced stage of malignancy. Also, overall survival depends on many factors, including response to adjuvant treatments.

There are few strengths of this study. First, it showed the safety of EUS-GE using EPASS in a high-risk group of patients with ascites and malignant GOO. Any procedural AE in such patients is serious and life-threatening. Second, this study also highlighted the various technical challenges encountered in detail, despite using EPASS. Third, we systematically followed all the patients at regular intervals until death or last contact to assess the impact of the procedure. The limitations of the study include the relatively small sample size, which was mainly due to the rarity of an evolving procedure from a single center. The second limitation was the retrospective analysis, although from prospectively maintained data. We made sure to document all clinical details systematically during the study period. Third, a control arm of other techniques was lacking to clearly establish whether EPASS was better and safer. However, it may be appropriate to say that fixing the jejunal segment to maintain close opposition with the stomach using EPASS made the procedure relatively safer. Future prospective multicenter studies comparing different techniques with large sample sizes are needed to evaluate to the technical success and sustained clinical benefit of the procedure in patients with ascites.

\section{Conclusions}

Ascites is common in patients with malignant GOO. EUS-GE is feasible in the presence of ascites with EPASS. The technical and clinical success rates were similar to those in patients without ascites.

\section{Competing interests}

The authors declare that they have no conflict of interest.

\section{References}

[1] Binmoeller KB, Shah J. Endoscopic ultrasound-guided gastroenterostomy using novel tools designed for transluminal therapy: a porcine study. Endoscopy 2012; 44: 499-503

[2] Itoi T, Itokawa F, Uraoka T et al. Novel EUS-guided gastrojejunostomy technique using a new double-balloon enteric tube and lumen-apposing metal stent (with videos). Gastrointest Endosc 2013; 78: 934939

[3] Khashab MA, Kumbhari V, Grimm IS et al. EUS-guided gastroenterostomy: The first U.S. clinical experience (with video). Gastrointest Endosc 2015; 82: 932-938

[4] Tyberg A, Perez-Miranda M, Sanchez-Ocana R et al. Endoscopic ultrasound-guided gastrojejunostomy with a lumen-apposing metal stent: A multicenter, international experience. Endosc Int Open 2016; 4: E276-E281

[5] Marrache MK, Itani MI, Farha J et al. Endoscopic gastrointestinal anastomosis: a review of established techniques. Gastrointest Endosc 2021; 93: 34-46

[6] Moore KP, Wong F, Gines P et al. The management of ascites in cirrhosis: Report on the consensus conference of the International Ascites Club. Hepatology 2003; 38: 258-266

[7] Chavan R, Ramchandani M, Nabi Z et al. Luminal and extraluminal bleeding during EUS-guided double-balloon-occluded gastrojejunostomy bypass in benign gastric outlet obstruction with portal hypertension. Video GIE 2019; 5: 64-67

[8] Dormann A, Meisner S, Verin N et al. Self-expanding metal stents for gastroduodenal malignancies: Systematic review of their clinical effectiveness. Endoscopy 2004; 36: 543-550

[9] Ge PS, Young JY, William Dong W et al. EUS-guided gastroenterostomy versus enteral stent placement for palliation of malignant gastric outlet obstruction. Surg Endosc 2019; 33: 3404-3411

[10] Itoi T, Ishii K, Ikeuchi N et al. Prospective evaluation of endoscopic ultrasonography-guided double-balloon-occluded gastrojejunostomy bypass (EPASS) for malignant gastric outlet obstruction. Gut 2016; 65: $193-195$

[11] Chen YI, Kunda R, Storm AC et al. EUS-guided gastroenterostomy: a multicenter study comparing the direct and balloon-assisted techniques. Gastrointest Endosc 2018; 87: 1215-1221

[12] Irani S. Placing a lumen-apposing metal stent desite ascites: feasibility and safety. Video GIE 2020; 5: 586-590 